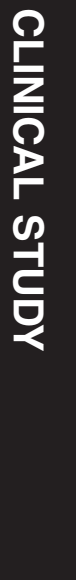

${ }^{1}$ Department of Anaesthesiology, Perioperative and Pain Medicine (SAMPE), Hospital de Clínicas de Porto Alegre (HCPA), Porto Alegre, Brazil

${ }^{2}$ Universidade Federal do Rio Grande do Sul (UFRGS), Porto Alegre, Brazil

${ }^{3}$ Department of Internal Medicine, Division of Cardiology, Hospital de Clínicas de Porto Alegre (HCPA), Porto Alegre, Brazil

${ }^{4}$ Post - Graduate Program in Cardiology, School of Medicine, UFRGS, Porto Alegre, Brazil

${ }^{5}$ Institute for Health Technology Assessment (IATS), HCPA, Porto Alegre, Brazil

Correspondence: C Alboim, Hospital de Clínicas de Porto AlegreService of Anesthesiology, Rua Ramiro Barcelos, 2350, 13th floor, Porto Alegre, RS 90035-903, Brazil Tel: +55 519678 4628; Fax: +55 5130120575 . E-mail: calboim@ hotmail.com

Received: 9 November 2015 Accepted in revised form: 15 August 2016 Published online: 16 September 2016

\section{The impact of preoperative evaluation on perioperative events in patients undergoing cataract surgery: a cohort study}

C Alboim², RB Kliemann², LE Soares², MM Ferreira', CA Polanczyk ${ }^{3,4,5}$ and A Biolo ${ }^{3,4,5}$

\begin{abstract}
Purpose Ambulatory surgery is a major area of surgical and anesthetic practice, and preoperative clinics are being increasingly used for low-risk surgical procedures. This study investigated the impact of preoperative evaluation on perioperative events in patients undergoing cataract surgery.
\end{abstract}

Methods This was a retrospective cohort study of 968 consecutive patients undergoing cataract surgery. Details of medical conditions, surgical, anesthetic, and postoperative information were collected from medical records. A logistic regression model was developed using propensity score adjustment for baseline characteristics. Results Out 968 patients included, 240 (24.7\%) underwent outpatient preoperative evaluation. There were no perioperative major cardiovascular events. Hypertension occurred in $319(33 \%)$ patients, accounting for $79.7 \%$ of all adverse events. Preoperative evaluation resulted in a lower hypertension rate after adjustment for propensity score $(\mathrm{OR}=0.6 ; 95 \%$ CI $0.41-0.93)$; no effects were observed on posterior capsule rupture and emergency visits/hospitalization within 7 days of surgery. Eighty-nine patients $(9.3 \%)$ had an initial systolic pressure $\geq 180 \mathrm{~mm} \mathrm{Hg}$, which was not associated with higher risk of posterior capsule rupture $(P=0.158)$ or postoperative adverse events $(P=0.902)$. Median waiting time to surgery was 6 and 2 months for evaluated and non-evaluated patients, respectively $(P<0.001)$.

Conclusions In the context of low-risk surgery and no major perioperative and postoperative outcomes, it appears that outpatient preoperative evaluation has no role in reducing adverse events in cataract surgery candidates. Despite fewer hypertensive episodes observed in evaluated patients, these episodes were not associated with any medical or surgical outcomes.

Eye (2016) 30, 1614-1622; doi:10.1038/eye.2016.203; published online 16 September 2016

\section{Introduction}

It is estimated that 200 million noncardiac surgical procedures are performed worldwide each year. ${ }^{1}$ Despite advances in perioperative care, about 3-5 million patients have vascular complications and up to 1 million die within 30 days of surgery. ${ }^{2}$ The risk, however, varies according to the type of surgery and patient comorbidities, reaching up to $11 \%$ in high-risk patients undergoing major surgical procedures. ${ }^{3}$ Aiming to prepare these patients and reduce perioperative complications, preoperative evaluation has become a common practice in recent years. ${ }^{4}$ Previous studies show a great variability in the forwarding rate (10 to $40 \%)^{5-7}$ and, despite of the presumed benefits for intermediate-to-high risks patients, the factors that determine whether an individual does or does not undergo preoperative evaluation remain unclear.

Ambulatory surgery is currently one of the major areas of surgical and anesthetic practice, with about $70 \%$ of procedures performed in the outpatient setting. ${ }^{8}$

Consequently, patients with multiple diseases are also being treated in the ambulatory surgery environment. ${ }^{9}$ In this context, preoperative assessment clinics are being increasingly used for patients who will undergo minor surgery, often to the detriment of candidates for major surgery. ${ }^{10,11}$ Among cataract surgery candidates, for instance, despite the low incidence of perioperative morbidity and mortality ${ }^{12,13}$ and because lack of recommendation for routine 
medical testing before surgery, ${ }^{14}$ these patients have been seen more frequently in preoperative consultations, and the referrals do not seem to be based on medical factors, but geographic variation. ${ }^{15}$

Since 2006, operates at our institution the preoperative clinic attended by anesthesiologists and referral may be performed as deemed necessary by the surgeon. Patients are referred with most routine tests already ordered by the surgeon (as the habit of the surgeon request it to all their patients is very present in our hospital), even in lowrisk surgery. Complementary tests may be requested during preoperative evaluation as it is needed.

In low-risk surgery, few studies have investigated the effect of preoperative consultations on perioperative care and patient outcomes, and the cost-effectiveness of these clinics has been questioned. ${ }^{16}$ We hypothesized that preoperative assessment would not be associated with intraoperative and postoperative adverse events in this population. The current study was therefore designed to evaluate the impact of outpatient preoperative evaluation on clinical and surgical perioperative events in patients undergoing elective cataract surgery.

\section{Materials and methods}

\section{Statement of ethics}

We certify that all applicable institutional and governmental regulations concerning the ethical conduct of research involving medical record review were followed. The study was approved by the Research Ethics Committee of Hospital de Clínicas de Porto Alegre (protocol number 120-348) and conducted in accordance with the provisions of the Declaration of Helsinki. As this was a historical cohort study, the ethics committee waived the requirement for written informed consent.

\section{Patients}

This was a retrospective cohort study of all consecutive patients aged 50 years or over who underwent elective cataract surgery from June 2006 to December 2010 in a Brazilian tertiary public hospital (Hospital de Clínicas de Porto Alegre, Porto Alegre, Brazil). Patients with previous eye surgery or other concomitant surgical procedures were excluded.

\section{Outpatient preoperative evaluation}

Patients who were seen in a preoperative assessment clinic or in any other clinic aimed at preoperative evaluation, after a cataract diagnosis by the ophthalmologist and up to 12 months prior to surgery, were regarded as having undergone outpatient preoperative evaluation. At our institution, preoperative evaluation may be performed as deemed necessary by the surgeon, and focuses on the assessment of clinical predictors of increased perioperative cardiovascular risk and improvement in the patient's condition for surgery and anesthesia. Most patients are referred to the pre anesthetic clinc and a few can still be forwarded by the anaesthesiologist to the clinician or cardiologist.

\section{Data collection}

Data were collected by a standardized method of medical record review. The principal investigator and two previously trained medical students reviewed the patients' medical records and collected data. At every 50 records reviewed, one was randomly selected to be reviewed again for quality control of data collection.

Details of medical conditions and comorbidities were obtained by reviewing all records, mostly ophthalmic consultation and clinic visits (anaesthesiologist, internist, and cardiologist). If the patient had not been seen by any of these specialists, data records from other medical specialties were thoroughly reviewed for information relevant to the study. History of ischemic heart disease, heart failure, stroke, creatinine values, and insulin-dependent diabetes were used to calculate the Revised Cardiac Risk Index (RCRI) proposed by Lee et al. ${ }^{17}$ Patients' medical records were also reviewed to relate each test performed preoperatively to the requesting team to confirm that all tests were ordered as part of the preoperative investigation. Tests ordered for reasons other than preoperative evaluation were excluded from the analysis. Waiting time for surgery was defined as the number of days between surgical indication by the ophthalmologist and surgery.

The intraoperative data collected included the American Society of Anesthesiologists (ASA) physical status classification, ${ }^{9}$ surgical technique, type of anesthesia, and vital signs. Clinical and surgical adverse events analyzed in this study, along with their definition, are described in Table 1. Myocardial infarction or ischemia, decompensated congestive heart failure, stroke or transient ischemic attack were considered major adverse cardiovascular events. Posterior capsule rupture and endophthalmitis were regarded as surgical outcomes, because they are considered, respectively, the most frequent and severe complications of cataract surgery. Postoperative adverse events were determined by reviewing the records of ophthalmologic follow-up evaluation performed within 7 days of surgery. Data on emergency visits or hospitalization during this 7-day period were also collected. In case of lack of postoperative information, investigators contacted patients by telephone to obtain the missing data. In the analysis of postoperative 
Table 1 Definition of adverse events

\begin{tabular}{|c|c|}
\hline Event & Definition \\
\hline Hypertension & $\begin{array}{l}\text { Increase in systolic pressure to } \geq 180 \mathrm{~mm} \mathrm{Hg} \text { or in diastolic pressure to } \geq 110 \mathrm{~mm} \mathrm{Hg} \text {, and/or } \\
\text { treatment with antihypertensive drugs from entrance into the operating room to discharge }\end{array}$ \\
\hline Arrhythmia & $\begin{array}{l}\text { Worsening of or new arrhythmia during the procedure, as informed by the anesthesiologist, or in } \\
\text { the recovery room, requiring new treatment or change in current treatment }\end{array}$ \\
\hline Bronchospasm & $\begin{array}{l}\text { Wheezing, dyspnea, coughing, or desaturation during the procedure, as informed by the } \\
\text { anaesthesiologist, or in the recovery room, requiring bronchodilator or theophylline }\end{array}$ \\
\hline Transient ischemic attack or stroke & $\begin{array}{l}\text { Abrupt onset of focal neurological deficit during the procedure, as informed by the } \\
\text { anaesthesiologist, or in the recovery room }\end{array}$ \\
\hline Myocardial ischemia & $\begin{array}{l}\text { New or more severe chest pain, followed by ischemic changes on ECG during the procedure, as } \\
\text { informed by the anaesthesiologist, or in the recovery room, requiring treatment }\end{array}$ \\
\hline Myocardial infarction & $\begin{array}{l}\text { New chest pain, ECG changes (changes in the ST segment, new Q wave) and enzyme changes } \\
\text { requiring treatment or ischemic symptoms with new bundle branch block on ECG, as informed } \\
\text { by the anaesthesiologist, or in the recovery room }\end{array}$ \\
\hline 7-day emergency/hospitalization & Admission to the emergency room or hospitalization within 7 days of surgery \\
\hline Posterior capsule rupture & $\begin{array}{l}\text { Operative report or ophthalmologic follow-up records containing this diagnosis or the report of } \\
\text { vitrectomy during cataract surgery }\end{array}$ \\
\hline Endophthalmitis & Medical record containing this diagnosis \\
\hline
\end{tabular}

Abbreviation: ECG, electrocardiogram.

events, initial systolic blood pressure $\geq 180 \mathrm{~mm} \mathrm{Hg}$, ASA physical status $\geq 3$ and RCRI $\geq 2$ were used as variables to predict adverse events.

\section{Outcome measures and sample size}

The primary end point with respect to the efficacy of outpatient preoperative evaluation in preventing intraoperative and postoperative adverse events in patients undergoing elective cataract surgery was the difference in the incidence of adverse events between evaluated and non-evaluated patients. On the basis of an expected incidence of total adverse events of $9 \%$ in the non-evaluated group, a sample size of 884 patients (221 in the evaluated group and 663 in the non-evaluated group) would be necessary to detect a difference as small as $2 \%$ ( $7 \%$ in the evaluated group), with a power of $80 \%$ and a significance level of 5\%. ${ }^{18,19}$ Sample size was calculated using WinPepi software, version $11.43 .^{20}$

\section{Statistical analysis}

Proportions of baseline characteristics were calculated for patients with and without outpatient preoperative evaluation. Data are expressed as number (\%) of patients for categorical variables, and as mean (SD) (if normally distributed) or median (interquartile range (IQR)) for continuous variables. Pearson's $\chi^{2}$-test was used to compare categorical variables. Results for normally distributed continuous variables were compared using Student $t$-tests, and results for non-normally distributed continuous variables were compared using the MannWhitney $U$-test. A $P$-value $<0.05$ indicated statistical significance. Statistical analysis was performed using SPSS, version 22.0 (IBM Corp., Armonk, NY, USA).

Univariate logistic regression analysis was performed for each adverse event. Then, because the groups differed from each other, a logistic regression model was developed using propensity score adjustment for baseline characteristics that could influence the likelihood of patients being referred for preoperative evaluation. Variables with a $P$-value $<0.10$ in the univariate analysis (age, sex, hypertension, coronary artery disease, congestive heart failure, arrhythmia, history of stroke/ transient ischemic attack, diabetes, creatinine $\geq 2 \mathrm{mg} / \mathrm{dl}$, and pulmonary disease) were used to develop a propensity score model in order to obtain adjusted odd ratios (ORs) with 95\% confidence intervals (95\% CIs). Multivariate logistic regression analysis was also performed to investigate the effect of initial systolic pressure $\geq 180 \mathrm{~mm} \mathrm{Hg}$ on posterior capsule rupture. The variables included in the model were propensity score, surgical technique (phacoemulsification or extracapsular cataract extraction), and glaucoma.

\section{Results}

A total of 1407 cataract operations performed between 2006 and 2010 were reviewed, 968 patients were included in the study and 439 were excluded because first eye had been previously operated. Of the 968 patients included, 240 were evaluated preoperatively and 728 went directly 
Table 2 Baseline characteristics of patients

\begin{tabular}{|c|c|c|c|}
\hline & \multicolumn{2}{|c|}{ Outpatient preoperative evaluation } & \multirow[t]{2}{*}{ P-value } \\
\hline & $Y E S(\mathrm{n}=240)$ & $N O(\mathrm{n}=728)$ & \\
\hline Age (years) ${ }^{\mathrm{a}}$ & $73(67.0-78.5)$ & $70(62.0-76.0)$ & 0.001 \\
\hline Weight $(\mathrm{kg})^{\mathrm{b}}$ & $69.4 \pm 13.3$ & $69.1 \pm 13.1$ & 0.749 \\
\hline Female sex & $135(56.3)$ & $457(62.8)$ & 0.072 \\
\hline \multicolumn{4}{|l|}{ Surgical technique } \\
\hline Extracapsular cataract extraction & $21(8.8)$ & $56(7.7)$ & 0.592 \\
\hline Phacoemulsification & $217(91.2)$ & $671(92.3)$ & \\
\hline \multicolumn{4}{|l|}{ Anesthetic technique } \\
\hline Peribulbar block & $233(97.0)$ & $718(98.6)$ & 0.235 \\
\hline General anesthesia & $6(2.5)$ & $10(1.4)$ & \\
\hline \multicolumn{4}{|l|}{ Prexisting comorbidities } \\
\hline Arrhythmia & $38(15.8)$ & $40(5.5)$ & 0.001 \\
\hline Congestive heart failure & $27(11.3)$ & $9(1.2)$ & 0.001 \\
\hline Coronary artery disease & $66(27.5)$ & $31(4.3)$ & 0.001 \\
\hline Creatinine $\geq 2 \mathrm{mg} / \mathrm{dl}$ & $17(7.7)$ & $15(2.5)$ & 0.001 \\
\hline Diabetes & $98(40.8)$ & $134(18.4)$ & 0.001 \\
\hline Dialysis & $8(3.6)$ & 0 & 0.001 \\
\hline Glaucoma & $19(7.9)$ & $53(7.2)$ & 0.679 \\
\hline History of stroke/TIA & $23(9.6)$ & $9(1.2)$ & 0.001 \\
\hline Hypertension & $188(78.3)$ & $419(57.5)$ & 0.001 \\
\hline Insulin use & $38(15.8)$ & $33(4.5)$ & 0.001 \\
\hline Pulmonary disease & $36(15.0)$ & $69(9.4)$ & 0.059 \\
\hline Smoking & $42(17.5)$ & $126(17.3)$ & 0.946 \\
\hline RCRI score & & & 0.001 \\
\hline 0 & $107(48.2)$ & $553(87.8)^{\mathrm{c}}$ & \\
\hline 1 & $73(32.9)^{c}$ & $66(10.9)$ & \\
\hline 2 & $36(16.2)^{c}$ & $7(1.2)$ & \\
\hline 3 & $6(2.7)^{c}$ & $1(0.2)$ & \\
\hline ASA physical status classification & & & 0.001 \\
\hline I & 0 & $68(9.4)^{c}$ & \\
\hline II & $156(65.0)$ & $605(83.3)$ & \\
\hline III & $84(35.0)^{c}$ & $52(7.2)$ & \\
\hline IV & 0 & $1(0.1)$ & \\
\hline
\end{tabular}

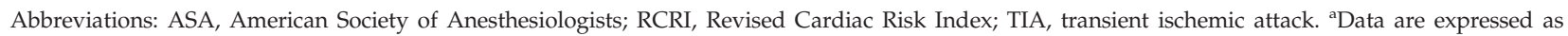
median (interquartile range). ${ }^{b}$ Data are expressed as mean (SD). ${ }^{c} P$-value $\leq 0.05$.

to surgery (Supplementary Figure). Phacoemulsification was performed in $888(91.7 \%)$ patients. There were no missing data for the presence of adverse events (yes/no) until discharge, and $97.5 \%$ of patients had information available on the variables of interest from the immediate 7-day postoperative period.

The baseline characteristics of the study sample are described in Table 2. Of 968 participants, 240 (24.7\%) underwent outpatient preoperative evaluation. Of these, $83.3 \%$ were seen by an anaesthesiologist, $12.5 \%$ by an internist, and $12.9 \%$ by a cardiologist. Only twenty four patients $(10 \%)$ were seen by more than one provider. The median waiting time between surgical indication and surgery was 6 months (IQR, 4-9 months) for outpatient- evaluated patients and 2 months (IQR, 1-3 months) for non-evaluated patients $(P<0.001)$.

Almost all cardiovascular risk factors (age, hypertension, coronary artery disease, congestive heart failure, arrhythmia, stroke, diabetes, and creatinine $\geq 2 \mathrm{mg} / \mathrm{dl}$ ) were more prevalent in outpatient-evaluated patients (Table 2). In the evaluated group, there were no ASA I patients, and only $16.2 \%$ of patients had an RCRI score of 0 . More than $80 \%$ of outpatientevaluated patients had an RCRI score of 2-3 and 62\% of patients were ASA III. There was no difference between the evaluated and non-evaluated groups regarding sex, pulmonary disease, glaucoma, or surgical technique. 
Table 3 Ordering of preoperative tests

\begin{tabular}{lccr}
\hline Tests & \multicolumn{2}{c}{$\begin{array}{c}\text { Outpatient preoperative } \\
\text { evaluation }\end{array}$} & P-value \\
\cline { 2 - 3 } & $Y E S^{\mathrm{a}}(\mathrm{n}=240)$ & $N O^{\mathrm{b}}(\mathrm{n}=728)$ & \\
\hline Complete blood count & $212(88.3)$ & $608(83.5)$ & 0.072 \\
ECG & $211(87.9)$ & $620(85.3)$ & 0.289 \\
Creatinine & $210(87.5)$ & $595(81.7)$ & 0.038 \\
Glucose & $180(75.0)$ & $480(65.7)$ & 0.008 \\
Chest X-ray & $204(85.0)$ & $588(80.8)$ & 0.141 \\
Exercise ECG & $8(3.3)$ & 0 & $<0.001$ \\
Myocardial scanning & $14(5.8)$ & 0 & $<0.001$ \\
Echocardiography & $13(5.4)$ & 0 & $<0.001$ \\
\hline
\end{tabular}

Abbreviation: ECG, electrocardiogram.

Data are expressed as $n(\%)$. ${ }^{a}$ Most tests were ordered by ophthalmologists.

${ }^{b}$ All tests were ordered by ophthalmologists.

Table 4 Preoperative optimization during outpatient preoperative evaluation

\begin{tabular}{lcc}
\hline Preoperative optimization strategy $^{\mathrm{a}}$ & $\mathrm{n}(36)$ & $\%$ \\
\hline $\begin{array}{l}\text { Drug therapy } \\
\text { ACE inhibitors, calcium channel blocker, }\end{array}$ & 10 & 31.25 \\
angiotensin-receptor blocker & 7 & 21.2 \\
ק-blocker & 4 & 12.5 \\
Diuretics & 6 & 18.7 \\
Anticoagulation reversal & 4 & 12.5 \\
Insulin or metformin & 4 & 12.5 \\
Others & & \\
& & \\
Procedures & 1 & 3.0 \\
Pacemaker & 0 & 0 \\
Coronary angioplasty/CABG & \\
\hline
\end{tabular}

Abbreviations: $\mathrm{ACE}$, angiotensin converting enzyme; $\mathrm{CABG}$, coronary artery bypass grafting. ${ }^{a} 36$ preoperative optimization strategies were used

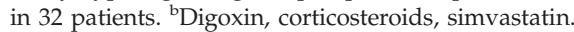

Overall, in $>90 \%$ of patients, at least one preoperative chest X-ray, electrocardiogram (ECG) or laboratory test was ordered, and these tests were mainly ordered by ophthalmologists. There was no difference between groups regarding the total number of tests ordered before surgery, except for creatinine, and glucose (Table 3). Additional cardiac stress testing (exercise ECG, myocardial scanning, and echocardiography) was ordered for $30(12.5 \%)$ patients in the outpatientevaluated group. Of these, six patients $(20 \%)$ had abnormal test results (myocardial ischemia, left ventricular ejection fraction $<40 \%$, and metabolic equivalents in exercise testing $\leq 2$ ); however, four of these patients had no changes in cardiovascular treatment despite abnormal findings.

Changes in clinical management during outpatient preoperative evaluation are shown in Table 4. Adjustment of drug therapy was performed in 33 (13.7\%) patients, and the most common was antihypertensive drug management $(42.2 \%)$. Changes in cardiovascular treatment (antihypertensive agents, $\beta$-blockers, and digoxin) were observed in $63 \%$ of patients for whom no cardiac stress test was ordered and in $36 \%$ of patients for whom at least one cardiac stress test was ordered $(P=0.054)$.

No major adverse cardiovascular events were reported in either group. Overall, hypertension was observed in 319 (33\%) patients, accounting for $79.7 \%$ of all adverse events. Minor intraoperative adverse events occurred in $355(36.7 \%)$ patients (Table 5).

In the univariate analysis, there was no difference between groups in the cumulative rate of medical and surgical events: $33.8 \%$ in outpatient-evaluated patients $v$ s

Table 5 Incidence of medical and surgical adverse events in cataract surgery

\begin{tabular}{|c|c|c|c|c|c|}
\hline & \multicolumn{3}{|c|}{ Outpatient preoperative evaluation } & \multirow[t]{2}{*}{ Crude OR $(95 \% \mathrm{CI})$} & \multirow[t]{2}{*}{ Adjusted OR $(95 \% \text { CI })^{\mathrm{a}}$} \\
\hline & Total $\mathrm{n}=968$ & $Y E S(\mathrm{n}=240)$ & $N O(\mathrm{n}=728)$ & & \\
\hline Perioperative event & $355(36.7)$ & $80(33.8)$ & $275(38.6)$ & $0.8(0.59-1.10)$ & $0.6(0.42-0.93)$ \\
\hline \multicolumn{6}{|l|}{ Clinical } \\
\hline Hypertension & $319(33)$ & $71(29.7)$ & $248(34.0)$ & $0.8(0.59-1.11)$ & $0.6(0.41-0.93)$ \\
\hline Bronchospasm & $1(0.1)$ & 0 & $1(0.1)$ & - & - \\
\hline Arrhythmia & 0 & 0 & 0 & - & - \\
\hline MACE & 0 & 0 & 0 & - & - \\
\hline \multicolumn{6}{|l|}{ Ophthalmic } \\
\hline Posterior capsule rupture & $70(7.4)$ & $22(9.2)$ & $48(6.6)$ & $1.4(0.83-2.39)$ & $1.1(0.55-2.27)$ \\
\hline Endophthalmitis & $5(0.7)$ & $1(0.4)$ & $4(0.6)$ & $1.3(0.14-11.7)$ & $1.7(0.14-20.1)$ \\
\hline \multicolumn{6}{|l|}{ Postoperative event } \\
\hline 7-day emergency/hospitalization & $12(1.3)$ & $3(1.3)$ & $9(1.3)$ & $1.0(0.27-3.78)$ & $0.8(0.14-4.71)$ \\
\hline
\end{tabular}

Abbreviations: MACE, major adverse cardiovascular events; OR, odds ratio.

There was more than one event per patient. ${ }^{a}$ Covariate adjustment using the propensity score (age, sex, hypertension, coronary artery disease, congestive heart failure, arrhythmia, history of stroke/transient ischemic attack, diabetes, creatinine $\geq 2 \mathrm{mg} / \mathrm{dl}$, and pulmonary disease). 
$38.6 \%$ in non-evaluated patients $(P=0.181)$. When adjusted for propensity score, a lower rate of events was found in the outpatient-evaluated group (OR $=0.6 ; 95 \%$ CI $0.42-0.93)$, which was mostly driven by the most frequent event, hypertension. The second most common adverse event was posterior capsule rupture, with an overall incidence of $7.4 \%$, accounting for $17.5 \%$ of all complications. There was no difference in the incidence of posterior capsule rupture between outpatient-evaluated and non-evaluated patients, even after adjustment $(\mathrm{OR}=1.1$; 95\% CI 0.55-2.27).

Twelve patients $(1.3 \%)$ visited the emergency department or were hospitalized in the immediate 7-day postoperative period. Of these, seven cases were surgical complications ( 3 endophthalmitis, 4 other causes) and five were clinical events ( 2 hypertension, 1 mental confusion, 1 pulmonary cause, 1 anticoagulation control). There was no difference between groups $(\mathrm{OR}=0.8 ; 95 \% \mathrm{CI} 0.14-4.71$ ) in the 7-day postoperative period, and none of these cases was related to major cardiovascular events. ASA III physical status appeared to increase the risk of emergency visits/hospitalization within 7 days of surgery compared with ASA I and II (OR=4.4; 95\% CI 1.39-14.24). RCRI $\geq 2$ was not associated with perioperative hypertension $(\mathrm{OR}=1.4 ; 95 \% \mathrm{CI} 0.79-2.57)$, posterior capsule rupture $(\mathrm{OR}=2.1$; 95\% CI 0.92-4.99), or hospitalization $(\mathrm{OR}=1.5$; 95\% CI 0.19-12.64).

Eighty-nine patients $(9.3 \%)$ had an initial systolic pressure $\geq 180 \mathrm{~mm} \mathrm{Hg}$. Logistic regression analysis showed no association between initial systolic pressure $\geq 180 \mathrm{~mm} \mathrm{Hg}$ and emergency visits/hospitalization within 7 days of surgery $(P=0.902)$ or with posterior capsule rupture $(P=0.158)$.

\section{Discussion}

In this historical cohort study, we evaluated patients who underwent cataract surgery with and without outpatient preoperative evaluation. Despite the presence of several comorbidities, there were no major cardiovascular events in our sample. Preoperative evaluation was associated with fewer hypertensive episodes, but these episodes were not associated with any medical or surgical outcome.

The incidence of major events in low-risk surgical procedures is very low, with a rate of $\sim 0.1 \%$ even in large studies. ${ }^{12-14}$ Because our sample consisted of almost 1000 individuals with absence of major events, a very large number of preoperative evaluations would be necessary to characterize differences in outcomes between evaluated and non-evaluated groups, and the expected benefit would be of questionable clinical significance. Despite the low incidence of major events, preoperative assessment for cataract surgery has increased in recent years, but this increase does not appear to be associated with clinical factors. ${ }^{15}$ Chen et al, ${ }^{21}$ using an observational cohort of Medicare beneficiaries undergoing cataract surgery, showed that the operating ophthalmologist was the strongest predictor of having a preoperative evaluation. ${ }^{21}$ In contrast, in the present study, referral for preoperative consultation was actually influenced by clinical factors. Patients with ASA I physical status and RCRI score of 0 , for example, were less frequently referred for preoperative consultation, with an inverse proportion at higher scores. However, these well-known predictors of perioperative risk could not be properly evaluated in the present study given the absence of major outcomes.

Routine preoperative testing occurred frequently in patients undergoing cataract surgery in the current study, since for $90 \%$ of patients at least one preoperative test was ordered mainly by ophthalmologists at the time of surgical indication, regardless forwarding to preoperative evaluation. Studies have demonstrated that hypertension, bronchospasm, and arrhythmia account for $>90 \%$ of intraoperative medical events in cataract surgery, and routine preoperative testing does not appear to predict their occurrence. ${ }^{22}$ Recent data indicate a reduction in the number of preoperative investigations when patients are evaluated in preoperative clinics. ${ }^{4}$ However, in the present study, most tests were ordered by the ophthalmologist before the patient was seen by a specialist for preoperative evaluation. Cardiac stress tests were ordered only for patients undergoing outpatient preoperative evaluation, and $9 \%$ of these patients had a cardiac stress test performed, a rate higher than that reported in the literature (0.8 to $2.4 \%$ in low-risk surgery). ${ }^{23}$ It is known that preoperative risk stratification with myocardial perfusion imaging provides an incremental prognostic value in intermediate-risk surgery, but not in low-risk operations. ${ }^{24}$ We believe that the association between clinical comorbidities and cataractogenesis, ${ }^{25}$ fear of cancellation of surgery, institutional requirements and medicolegal concerns may have contributed to the present results regarding the ordering of preoperative tests. ${ }^{26}$

Hypertension, a minor outcome, was the most common adverse event in the current study. Although previous studies ${ }^{14,18}$ have also described perioperative hypertension as the most common outcome in cataract surgery, its incidence was higher in our sample. This can be explained by two reasons: patients in developing countries have worse access to health care and consequently poorer control of their disease; and the criteria of hypertension, in which the presence of one measure systolic pressure $\geq 180 \mathrm{~mm} \mathrm{Hg}$ or dyastolic pressure $\geq 110 \mathrm{~mm} \mathrm{Hg}$, even if transient was considered hypertension. Moreover, in $28 \%$ of patients it was used 
intraoperative antihypertensive treatment, that was also considered, according to Table 1 . Our results suggest a beneficial effect of outpatient preoperative evaluation on hypertension control. However, initial systolic pressure $\geq 180 \mathrm{~mm} \mathrm{Hg}$ was not associated with perioperative adverse events. Phillips et al, ${ }^{19}$ showed that preanesthesia medical examination before ophthalmic surgery may have significant implications for the overall health of patients by identifying new and unstable medical conditions, although it does not commonly alter conduct of ophthalmic surgery or affect immediate postoperative outcomes. Thus, the cost-effectiveness of preoperative consultations has been questioned. ${ }^{16}$ Our data showed no influence of preoperative assessment on posterior capsule rupture, incidence of endophthalmitis, or emergency visits/hospitalization within 7 days of surgery.

One of the reasons for referring ophthalmic patients to preoperative evaluation is an attempt to decrease the rate of cancellations on the day of surgery. ${ }^{27}$ A Brazilian study reported that $\sim 21 \%$ of scheduled cataract operations are cancelled per year in the Brazilian public health system, and the main reason for cancellation is uncontrolled hypertension. ${ }^{28}$ The fact that, in the current study, hypertension was not related to major clinical events or to higher incidence of surgical events, even in patients with initial systolic pressure $\geq 180 \mathrm{~mm} \mathrm{Hg}$, leads us to question this reason for postponing cataract surgery.

Cataract is the leading cause of blindness worldwide, and the only effective treatment to restore vision is surgery. ${ }^{29}$ Significant improvement in quality of life has been observed after cataract surgery, and these beneficial effects extend to the performance of professional activities. ${ }^{30,31}$ Unfortunately, preoperative evaluation may delay this process, as demonstrated in the present study by the 4-month delay in surgery of patients referred for preoperative consultation. The fact that only $10 \%$ of evaluated patients were seen by more than 1 provider allows us to infer that this is not related to appropriate management of the patient's preexisting comorbidities but waiting time to consult. Service agility, integration of multidisciplinary teams and implementation of protocols are essential in the public health setting. This is particularly important in developing countries, where the demands for resources are significant but the available funds are limited. Considering that cataract surgery is a low-risk procedure, routine communication between ophthalmologists and primary care physicians may be more important to the overall care of the patient than concerns about surgical and medical perioperative complications.

This study has some limitations. First, it was conducted at a single tertiary referral center, which impacts the generalizability of the findings to other populations.
Second, because the study was designed as a historical cohort study (retrospectively analysis of prospectively gathered data), we relied on information from medical records, anesthesia records, and surgical reports provided by anaesthesiologists and ophthalmologists, thus subjective reporting bias may have occurred. In addition, information on cancellation of surgery was not available. Third, as patients with more severe clinical status were selectively referred for preoperative evaluation, one would expect an increase in the risk of adverse events (and even if the evaluation was beneficial, an effect might not have occurred), producing a type of bias known as confounding by indication. In the present study, this methodological issue was addressed using logistic regression analysis adjusted for propensity score. Finally, the low incidence of major cardiovascular events limited our conclusions about the difference between preoperatively evaluated and non-evaluated patients regarding these outcomes. Conversely, absence of these events in almost 1000 patients, in a 4-year period, is information of great importance to our institution.

In conclusion, preoperative assessment clinics have been implemented in recent years aiming to reduce perioperative adverse events. However, in the present study, in the surgical setting of low-risk procedures, no major clinical events occurred and surgical outcomes were not associated with specific clinical conditions. Patients undergoing outpatient preoperative evaluation were clinically managed, and this resulted in better perioperative blood pressure control. However, hypertension was not associated with any adverse event. Therefore, it was not possible to determine the benefit of outpatient preoperative evaluation in reducing adverse events in candidates for cataract surgery. We believe that priorities should be rearranged in order to optimize the resources of preoperative assessment for major surgical procedures and expand the access of cataract surgery candidates to primary health care.

\section{Summary}

\section{What was known before}

- Ambulatory surgery is a major area of surgical and anesthetic practice, and preoperative clinics are being increasingly used for low-risk surgical procedures, like cataract surgery.

- However, few studies have investigated the real effect of preoperative consultations on perioperative care and patient outcomes in this population.

\section{What this study adds}

- In the context of low-risk surgery and no major perioperative and postoperative outcomes, it appears that outpatient preoperative evaluation has no role in reducing adverse events in cataract surgery candidates. 


\section{Conflict of interest}

The authors declare no conflict of interest.

\section{Author contributions}

CA: study concept and design, conduction of the study project, execution and coordination of data collection, statistical analysis, and drafting of the article; final approval of the article. RBK: study concept and design, data collection and insertion into database; drafting of the article, final approval of the article. LES: study concept and design, data collection and insertion into database; drafting of the article, final approval of the article. MMF: study concept and design, data collection and insertion into database; drafting of the article, final approval of the article. CAP: conduction of the study project, statistical analysis, and drafting of the article; final approval of the article. AB: study concept and design, study advisor, statistical analysis, drafting and review of the article; final approval of the article.

\section{Acknowledgements}

We thank the translation agency Scientific Linguagem for assistance with the English-language presentation of the manuscript. The authors have no financial relationships relevant to this article to disclose.

\section{References}

1 Weiser TG, Regenbogen SE, Thompson KD, Haynes AB, Lipsitz SR, Berry WR et al. An estimation of the global volume of surgery: a modelling strategy based on available data. Lancet 2008; 372: 139-144.

2 Devereaux PJ, Chan MT, Alonso-Coello P, Walsh M, Berwanger O, Villar JC et al. Association between postoperative troponin levels and 30-day mortality among patients undergoing noncardiac surgery. JAMA 2012; 307: 2295-2304.

3 Fleisher LA, Fleischmann KE, Auerbach AD, Barnason SA, Beckman JA, Bozkurt B et al. 2014 ACC/AHA guideline on perioperative cardiovascular evaluation and management of patients undergoing noncardiac surgery: a report of the American College of Cardiology/American Heart Association Task Force on practice guidelines. J Am Coll Cardiol 2014; 64: e77-137.

4 Lee A, Kerridge RK, Chui PT, Chiu CH, Gin T. Perioperative Systems as a quality model of perioperative medicine and surgical care. Health Policy 2011; 102: 214-222.

5 Auerbach AD, Rasic MA, Sehgal N, Ide B, Stone B, Maselli J. Opportunity missed: medical consultation, resource use, and quality of care of patients undergoing major surgery. Arch Intern Med 2007; 167: 2338-2344.

6 Bugar JM, Ghali WA, Lemaire JB, Quan H. Utilization of a preoperative assessment clinic in a tertiary care centre. Clin Invest Med 2002; 25: 11-18.
7 Parker BM, Tetzlaff JE, Litaker DL, Maurer WG. Redefining the preoperative evaluation process and the role of the anesthesiologist. J Clin Anesth 2000; 12: 350-356.

8 Jarrett PE. Day care surgery. Eur J Anaesthesiol Suppl 2001; 23: 32-35.

9 Pasternak L. Risk Assessment in Ambulatory Surgery: challenges and new trends. Can J Anaesth 2004; 51: 12-16.

10 Hofer J, Chung E, Sweitzer BJ. Preanesthesia evaluation for ambulatory surgery: do we make a difference? Curr Opin Anaesthesiol 2013; 26: 669-676.

11 Thilen SR, Bryson CL, Reid RJ, Wijeysundera DN, Weaver EM, Treggiari MM. Patterns of preoperative consultation and surgical specialty in an integrated healthcare system. Anesthesiology 2013; 118: 1028-1037.

12 Mathis MR, Naughton NN, Shanks AM, Freundlich RE, Pannucci CJ, Chu Y et al. Patient selection for day case-eligible surgery: identifying those at high risk for major complications. Anesthesiology 2013; 119: 1310-1321.

13 Warner MA, Shields SE, Chute CG. Major morbidity and mortality within 1 month of ambulatory surgery and anesthesia. JAMA 1993; 270: 1437-1441.

14 Schein OD, Katz J, Bass EB, Tielsch JM, Lubomski LH, Feldman MA et al. The value of routine preoperative medical testing before cataract surgery. Study of Medical Testing for Cataract Surgery. N Engl J Med 2000; 342: 168-175.

15 Thilen SR, Treggiari MM, Lange JM, Lowy E, Weaver EM, Wijeysundera DN. Preoperative consultations for medicare patients undergoing cataract surgery. JAMA Intern Med 2014; 174: 380-388.

16 Newman MF, Mathew JP, Aronson S. The evolution of anesthesiology and perioperative medicine. Anesthesiology 2013; 118: 1005-1007.

17 Lee TH, Marcantonio ER, Mangione CM, Thomas EJ, Polanczyk CA, Cook EF et al. Derivation and prospective validation of a simple index for prediction of cardiac risk of major noncardiac surgery. Circulation 1999; 100: 1043-1049.

18 Lira RP, Nascimento MA, Moreira-Filho DC, Kara-Jose N, Arieta CE. Are routine preoperative medical tests needed with cataract surgery? Rev Panam Salud Publica 2001; 10: 13-17.

19 Phillips MB, Bendel RE, Crook JE, Diehl NN. Global health implications of preanesthesia medical examination for ophthalmic surgery. Anesthesiology 2013; 118: 1038-1045.

20 Abramson JH. WINPEPI update: computer programs for epidemiologists and their teaching potential. Epidemiol Perspect Innov 2011; 8: 1.

21 Chen CL, Lin GA, Bardach NS, Clay TH, Boscardin WJ, Gelb AW et al. Preoperative medical testing in Medicare patients undergoing cataract surgery. N Engl J Med 2015; 372: 1530-1538.

22 Keay L, Lindsley K, Tielsch J, Katz J, Schein O. Routine preoperative medical testing for cataract surgery. Cochrane Database Syst Rev 2012; 3: CD007293.

23 Kerr EA, Chen J, Sussman JB, Klamerus ML, Nallamothu BK. Stress testing before low-risk surgery: so many recommendations, so little overuse. JAMA Intern Med 2015; 175: 645-647.

24 Hashimoto J, Nakahara T, Bai J, Kitamura N, Kasamatsu T, Kubo A. Preoperative risk stratification with myocardial perfusion imaging in intermediate and low-risk non-cardiac surgery. Circ J 2007; 71: 1395-1400.

25 Nemet AY, Vinker S, Levartovsky S, Kaiserman I. Is cataract associated with cardiovascular morbidity? Eye (Lond) 2010; 24: $1352-1358$. 
26 Roizen MF. More preoperative assessment by physicians and less by laboratory tests. N Engl J Med 2000; 342 204-205.

27 Bamashmus M, Haider T, Al-Kershy R. Why is cataract surgery canceled? A retrospective evaluation. Eur J Ophthalmol 2010; 20: 101-105.

28 Magri MPF, Espíndola RF, Santhiago MR, Mercadante EF, Kara Júnior N. [Cancellation of cataract surgery in a public hospital]. Arq Bras Oftalmol 2012; 75: 333-336.
29 Snellingen T, Evans JR, Ravilla T, Foster A. Surgical interventions for age-related cataract. Cochrane Database Syst Rev 2002; (2): CD001323.

30 Kara-Junior N, Santhiago MR, Parede TRR, Espindola RF, Mazurek MGG, Germano R et al. [Influence of cataract surgical correction on working perception]. Arq Bras Oftalmol 2010; 73: 491-493.

31 Watkinson S, Seewoodhary R. Cataract management: effect on patients' quality of life. Nurs Stand 2015; 29: 42-48.

Supplementary Information accompanies this paper on Eye website (http://www.nature.com/eye) 